\title{
A Continues Double Auction Method for Resource Allocation in Economic Grids
}

\author{
Sanaz Teymouri \\ Department of Computer Engineering \\ Science and Research Branch, Islamic Azad \\ University \\ Tehran, Iran
}

\author{
Amir Masoud Rahmani \\ Department of Computer Engineering \\ Science and Research Branch, Islamic Azad \\ University \\ Tehran, Iran
}

\begin{abstract}
Sharing and optimized usage of resources utilities are one of the most essential goals in Grid systems. In such environments, patterns and aims of the resource providers and users for supply/demand differ. Beside, resource allocation algorithms attempt to provide the condition in which firstly providers are encouraged to keep on sharing their own computing resources and secondly the users are promoted to use the available resources to execute their jobs. Resource allocation ability to satisfy both sides, along with considering the users and providers mutual benefits, is a challenging task in grid market places.

By a method suggested in this paper Continuous Double Auction, which is one of the most used methods in grid marketing, is improved through updating bids by the auctioneer itself. Also in this paper a method is presented for the providers to determine the resource price based on their workload and for users to determine their bids based on jobs deadlines. The results show an improvement in both economic utilization and scheduling utilization compared to the standard Continuous Double Auction.
\end{abstract}

\section{Keywords}

Economic Grid, Resource Allocation, Double Auction.

\section{INTRODUCTION}

Grid systems provide a flexible and safe environment to share resources such as processors, memory, band width, software and licenses. In many organizations, there exist numerous idle resources. Grid computing creates a frame to utilize the idle resources and also increasing the resource usage. The users compete with each other for using these resources with lesser price and better quality. Also the providers compete for selling the resources in the highest price. The traditional resource allocation protocols, which require central control on their environment, are not suitable for economic grids, in which users and providers are distributed geographically. For resource management and allocation in such distributed environments, which the economic mutual benefits are of essential importance, the economic resource management models are used [1, 2, 3]. The most popular economic resource management models are: commodity market, Posted Price, Tendering/ Contact-Net, Auction, etc [1]. One of the most used economic models is Auction. There have been many studies on solving the resource allocation matters in grid $[4,5,6,7,8,9,10,11,12]$. In real world the auctions are used extendedly, especially for the sale of items in a certain period of time. The three main components in an auction are: resource provider, auctioneers (mediators) and users. The auction model supports one-to-many negotiation, between a provider (seller) and many consumers (buyers), and reduces negotiation to a single value. The auctioneer determines the policy of auction, acceptable for the consumers and the providers [1].

Auctions are divided into two main categories: 1- single-sided auction 2- double-sided auction. In single-sided auction only one side bids and the other side can only accept or reject. The four main types of single-sided auction are: English Auction, First Price Sealed Auction, Vickrey Auction (Second Price Sealed Auction) and Dutch Auction [13]. In the double-sided auctions both the seller and the buyer can send their bids and asks. There are three famous double-sided auctions: Call auction, CDA (Continuous Double Auction), Hybrid Auction [13]. In the CDA model, buy orders (bids) and sell orders (asks) can be submitted at anytime during the trading period. If at any time there are open bids and asks that match or are compatible in terms of price and requirements, a trade is executed instantly [1]. In this method the trade is done with the average price of ask and bid. One of the most significant subjects which matters to the users is when and how much to raise their bids to reach their desired resource on-time with a reasonable increased price. In this paper, the appropriate time and amount of user's bids for updating are determined by the auctioneer, which increases the jobs success rate and providers benefits immensely while decreases the users' cost. In this paper a method is presented for the providers to determine the resource price based on their workload and for users to determine their bids based on jobs deadlines, which provides the possibility to change the bid/ask in different auctions based on the situations of the participants in that auction.

\section{RELATED WORKS}

Adabi et al. [14] presented a new negotiation model for designing Market- and Behavior- driven Negotiation Agents that addressed computational grid resource allocation problem. This work introduced rational negotiation protocol and negotiation policy that modeled the effective factors used by negotiators of real-life trading market for making concession amount in negotiation process. 
Double auction models have been investigated by several researchers. Grosu and Das [15] presented the auction allocation model and three auction-based protocols: FirstPrice Auction, Vickrey Auction, and Double Auction. They studied them in terms of economic efficiency and system performance. The results showed that when they consider a mix of risk-averse and risk-neutral users First-Price Auction favors resources while Vickrey Auction favors users. On the other hand, the Double Auction favors both users and resources. Kant and Grosu [16] proposed a double auction allocation model for grids and evaluated three double auction protocols for resource allocation: Preston-McAfee Double Auction, Threshold Price Double Auction and CDA. The results showed that CDA protocol is better than both resource`s and user`s perspective providing high resource utilization. Tan and Gurd [13] proposed a stable continues double auction, based on the more conventional CDA. It alleviates the unnecessarily volatile behavior of the CDA, while maintaining other beneficial features. Assuncao and Buyya [17] presented a research on the communication requirements of First-Price sealed, English, Dutch, and Continuous double auctions for resource allocation in Grid computing environments. They showed English auctions present higher communication requirements while Continuous double auctions present least demand of communication. Izakian et al. [18] introduced a continuous double auction method in which resources are considered as provider agents and users as consumer agents. In each time step, each provider agent determines its requested value based on its workload and each consumer agent determines its bid value based on two constraints: the remaining time for bidding, and the remaining resources for bidding.

\section{THE RELATIONSHIP BETWEEN USERS/PROVIDERS AND AUCTIONEERS}

In the method presented in this paper, users and providers connect with some auctioneers randomly. In methods with a central system which saves the markets information, maintaining, updating and searching are challenging and timeconsuming, in this study it is considered that the information is distributed completely decentralized between the auctioneers and each auctioneer keeps the information of users and providers locally. Each user or provider can connect to several auctioneers to participate in different auctions to increase its chance. Figure 1 shows the structure of the relationship between users/providers and auctioneers. In case of success in an auction, the user will cancel its bid among other auctioneers. The bids stay with the auctioneer until they are canceled or succeed in a trade. The auctions are held in certain period of time and at the beginning of each period providers update their asks. When a provider connect with an auctioneer for the first time, determines how long its ask is valid and then it will be canceled. Users and providers may choose the auctioneers based on different parameters (distance, credit and popularity ...) which is not considered in this study, and the choosing of the auctioneers is considered random.

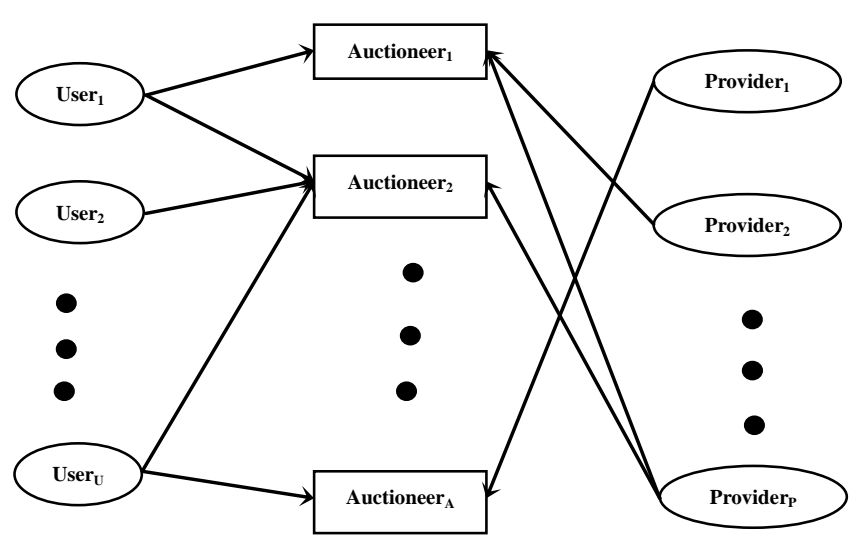

Figure 1: Structure of the relationship between users/providers and auctioneers

\section{DETERMINING ASKS AND BIDS}

In the real world buyers and sellers set a minimum and maximum price for the trade of goods or services. The buyer is aware of the fact that considering the market and the time, it is impossible to make a trade below a minimum cost (which is recognized by the buyer itself). Considering its budget and importance of the good or service for it, the buyer will not go above a self recognized maximum cost. On the other hand the seller is aware of the fact that considering the market, it cannot determine a price over a maximum cost for the trade (which is recognized by the seller itself). Considering its condition and the real value of the good or service, the seller will not go bellow a self recognized minimum cost. In the method presented in this paper, also this fact is considered for determining the users' bids and providers' asks for trades in the grid. Users, considering their understanding of market, set a minimum for their trades. This minimum here is shown by: $\mathrm{U}_{\min }$ and considering their condition they set a maximum which here is shown by: $U_{\max }$. These values can vary between different users. And so the amount of maximum and minimum for providers are named: $\mathrm{P}_{\max }$ and $\mathrm{P}_{\min }$ and can vary depending of their own condition and their understanding of market.

The users set their bids for executing each job unit something between $U_{\min }$ and $U_{\max }$ and in this pricing they consider jobs deadlines. Hence if the remaining time, till the starting of the job's execution is lesser, the bid for executing each unit of that job raises so that the chance of the job for reaching the resource increases. Here the deadline is the remaining time which before it the job must start executing, the execution time is not part of it and the important point is the time which the job have before it must start to execute. Providers set their asks for the execution of each job unit an amount between $\mathrm{P}_{\min }$ and $\mathrm{P}_{\max }$ and in this pricing they consider their workload. The more workload for the provider the more is its ask for a job unit execution. A resource here is a computer system with different abilities which is capable of running users' jobs during limited time. Here provider's workload means the number of job units that are waiting in a resource queue plus the remaining amount of the current job executing in the 
resource. The auctioneers are always aware of the providers' workload and users' job deadlines which they are connected to and can give the maximum of these amounts during a certain period of time to providers and users for determining bid and ask, with the names of MAX ${ }_{\text {work }}$ and MAX deadline. The amounts of $\mathrm{MAX}_{\mathrm{work}}$ and $\mathrm{MAX}_{\text {deadline }}$ in this period may increase gradually; still the updated amount is given to providers and users. In this way, the bids and asks which users and providers give to different auctioneers may vary; hence the price in different auctions may change locally depending on the condition of that auction. For example: In an auction where the $\mathrm{MAX}_{\text {work }}$ is 400 , a workload equal to 100 is more influential on raising the price compared to a similar auction where $\mathrm{MAX}_{\mathrm{work}}$ is equal to 600 . The Provider use formula 1 for determining the ask and users use formula 2 for determining the bid.

Ask $=\mathrm{P}_{\min }+\left(\right.$ workload $\left./ \mathrm{MAX}_{\text {work }}\right) *\left(\mathrm{P}_{\max }-\mathrm{P}_{\min }\right)$

$\mathrm{Bid}=\mathrm{U}_{\max }-\left(\right.$ deadline $\left./ \mathrm{MAX}_{\text {deadline }}\right) *\left(\mathrm{U}_{\max }-\mathrm{U}_{\min }\right)$

\section{UCDA METHOD}

Because of employing bid updating by auctioneer in CDA method, the method presented in this paper is named UCDA. Auctioneers are the mediators between users and providers for trades. They introduce suitable resources to users by receiving bids and asks and performing auctions. Providers send their asks for job units while users send their bids for job units, job length, deadline, $U_{\max }$ and $U_{\min }$ to the auctioneer. The providers/users can send their asks/bids to the auctioneer whenever during an auction period. The unsuccessful and valid bids are sorted descending and the valid asks are sorted ascending in two different lists. When a new bid arrives, it is compared with the first ask in asks list, if it was greater, the trade is made with the price equal to the average of bid and ask. Otherwise, the bid is added into the bids list in its proper place. When a new ask arrives, it is compared with the first bid in the bids list, if it was lesser a match happens and the trade is made. The new ask is added into to the ask list in its proper place and will stay in it until it is valid. The auctioneer must be aware of the resources release time at all times (the time of the exit of the last job from resource queue) so that while matching the bids and asks, it can check if the job can reach the resource before its deadline, if the job goes in the queue.

At the beginning of each auction period, providers update their asks considering their current workload at the related auctioneer according to formula 1 . Auctioneer receives the updated asks and resorts the ask list and compares asks from the beginning of the list with the bid list and does all possible trades related to each ask. Updating bids is done by the auctioneer itself and differently. To do so, a period named updating period is considered, which lasts half the period of the auction. Updating is done at the beginning of updating period- at the beginning and middle of each auction periodand during which all bids are updated considering their current remaining deadline and according to formula 2 , and bid list is resorted. Then from the beginning of the list, bids are compared to the ask list and all possible trades are done by the auctioneer, in case of trade the related bid is deleted from the list. Unlike the asks, the update of bids is done by the auctioneer, because of greater number of the bids and for simplicity and preventing many unnecessary relationship between the users and the auctioneers. Also since in auctions the proper time and amount of updating is a challenge for users, in UCDA these tasks are considered twice in each auction period and their amount is equal to the result of formula 2 .

\section{SIMULATION AND RESULTS}

Simulation for evaluating the presented method is done in Visual studio.Net and by C\# programming language. Table 1 shows the simulation parameters. In this simulation it is assumed that all providers' resources are computer systems with the ability if executing one job unit per time unit. Here the simulation is only done for a sole auctioneer and the users and providers connected to it locally, which can show the system's manners in general.

Table 1: Simulation Parameters

\begin{tabular}{|c|c|}
\hline Parameter & Value \\
\hline Users' Number & 25 \\
\hline Providers' Number & 10 \\
\hline Deadline & $\begin{array}{c}\text { In }[1 . .400] \text { with } \\
\text { uniform distribution }\end{array}$ \\
\hline Job Length & $\begin{array}{c}\text { In [1 .. 200] with } \\
\text { uniform distribution }\end{array}$ \\
\hline MAX $_{\text {work }}$ & 600 job units \\
\hline MAX $_{\text {deadline }}$ & 400 time units \\
\hline $\mathrm{U}_{\max }$ & $\begin{array}{c}\text { In [85 .. 95] with } \\
\text { uniform distribution }\end{array}$ \\
\hline $\mathrm{U}_{\min }$ & $\begin{array}{c}\text { In [75 .. 85] with } \\
\text { uniform distribution }\end{array}$ \\
\hline $\mathrm{P}_{\max }$ & $\begin{array}{l}\text { In }[90 . .100] \text { with } \\
\text { uniform distribution }\end{array}$ \\
\hline $\mathrm{P}_{\text {min }}$ & $\begin{array}{c}\text { In }[80 \text {.. 90] with } \\
\text { uniform distribution }\end{array}$ \\
\hline
\end{tabular}

Simulation runs during 100 periods, which each period is 3000 time units. Arrival time of jobs have Poisson distribution with the average of 10, 20 and 30 units of time. In 30, about 10000 jobs, in 20, about 15000 jobs and in 10 , about 30000 jobs are created. In this simulation the jobs waiting for execution in the resources are placed in a FIFO queue and are sent to the resource for executing one at a time. Providers register have uniform distribution with average of 20 units of time. Results shown in figures 2 to 7 are the average of 20 simulation runs. The results compare UCDA and CDA. In CDA asks and bids are determined randomly between $U_{\max }$ and $\mathrm{U}_{\min }$ for the users and the $\mathrm{P}_{\max }$ and $\mathrm{P}_{\min }$ for providers. Also asks are updated in the same way at the beginning of each period.

Updating bids by the auctioneer in updating periods and finishing all possible trades, increases jobs success rate noticeably. The success rate is the ratio of successful jobs to the total number of jobs. Figure 2 shows this increase. The jobs success rate in UCDA compared to CDA has increased 
by $58 \%$. In both methods the more jobs there is, the competition gets fiercer and the success rate is decreased.

Because of increased jobs success rate in UCDA compared to CDA, the average benefit of providers also increases which in figure 3 this is shown for different number of jobs. As the jobs increase the competition for the resources and number of trades are increased and the providers' benefit is increased. In UCDA the resources' idle time is lesser and figure 4 shows this. The reason for this decrease is the increase in jobs' success rate and therefore an increase in resources' amount of workload in UCDA. In both methods by increasing the number of jobs, the resources get busier and their idleness reduces.

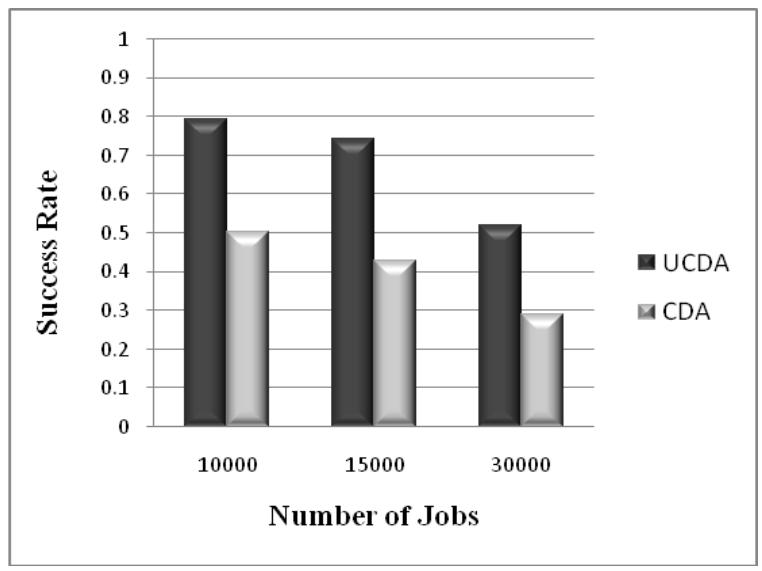

Figure 2: Increase of jobs' success rate

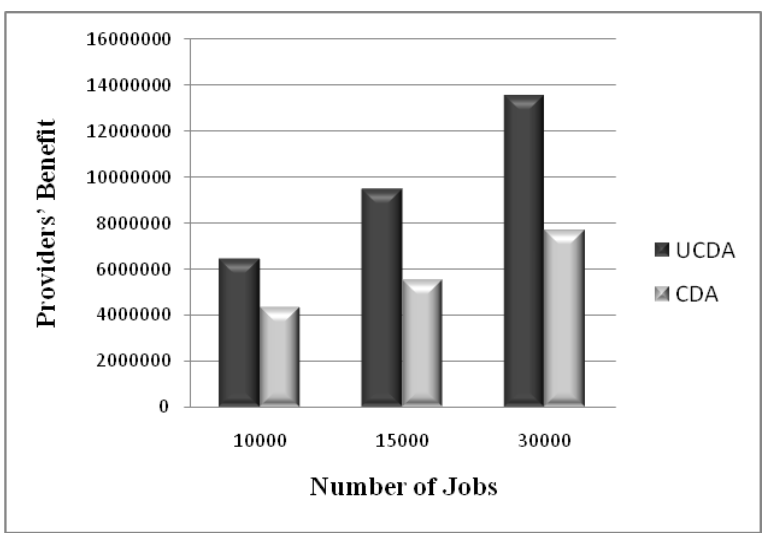

Figure 3: Increase of providers' benefit

According to figure 5 the users' average cost for the execution of a job unit in UCDA has decreased compared to CDA. The reason is the users' method for determining bids that is based on remaining job deadline and therefore the bids for jobs that have longer deadline are lessen which in random method this aspect is not considered. By increased jobs number and increased competition, the resources are allocated with higher price.

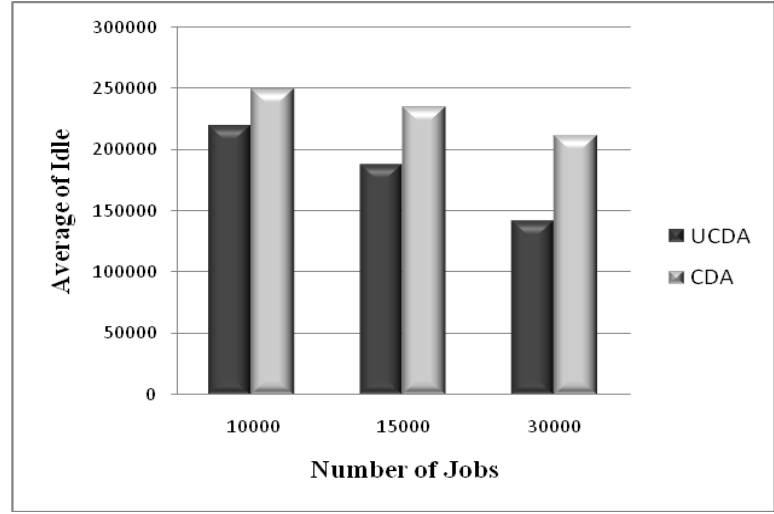

Figure 4: Decrease of providers' idleness

Though in UCDA jobs' success rate is higher and resources are busier, the average of jobs' response and waiting time haven't increased and according to figures 6 and 7 this average has decreased compared to CDA. The reason for this reduction could be the greater load balance in UCDA. It is normal in both methods that with increased jobs' number and busier resources the average of response and waiting time increases.

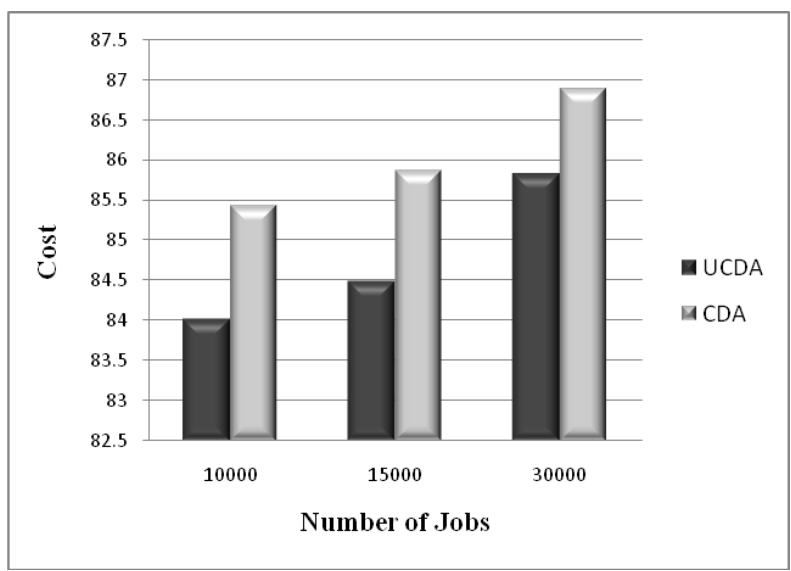

Figure 5: Decrease of users' average cost

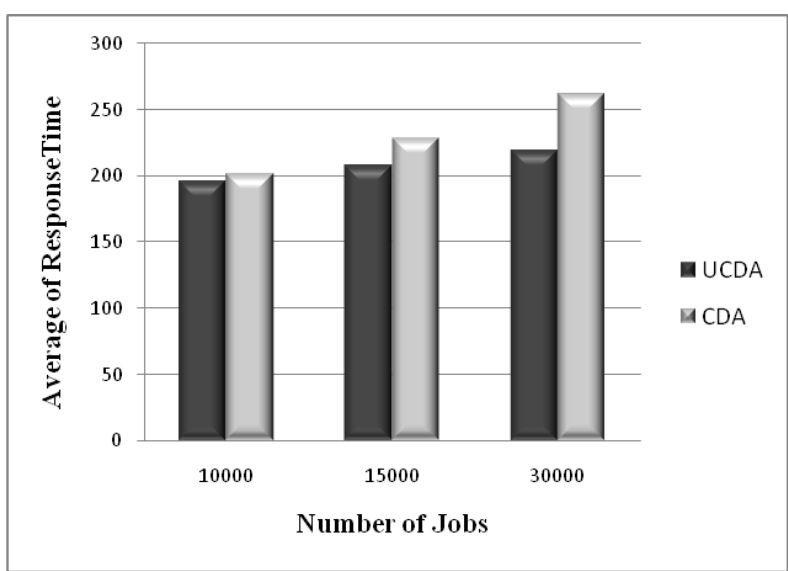

Figure 6: Decrease of average of response time

In 20 simulation runs for different number of jobs, providers' benefit balance in UCDA is greater compared to CDA. This is shown in figures 8, 9 and 10 for 10000, 15000 and 30000 jobs in three random runs. As the figures show, in UCDA, jobs and 
therefore the benefit of trades are distributed more balanced on the available resources. The reason is, more success jobs in CDA are allocated to cheaper resources but in UCDA, updating of bids leads to on-time increase and provides more distribution balance on all resources.

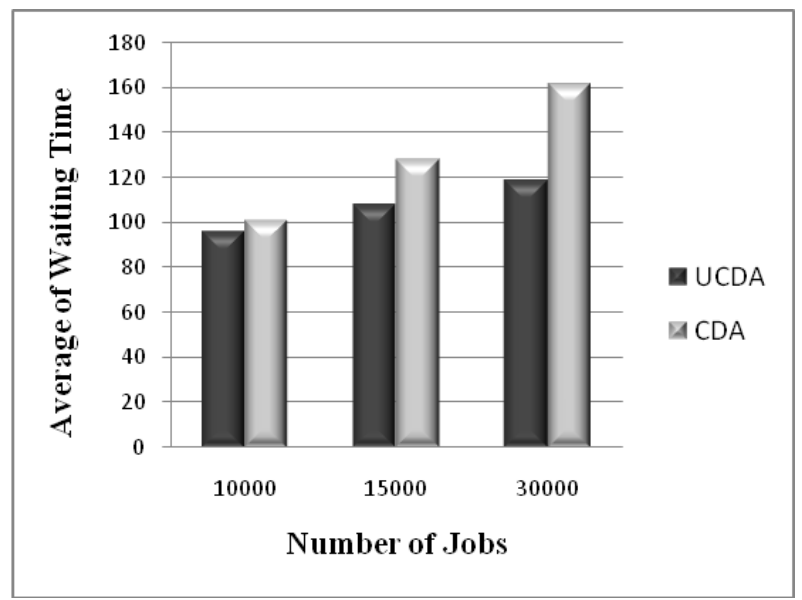

Figure 7: Decrease of average of waiting time

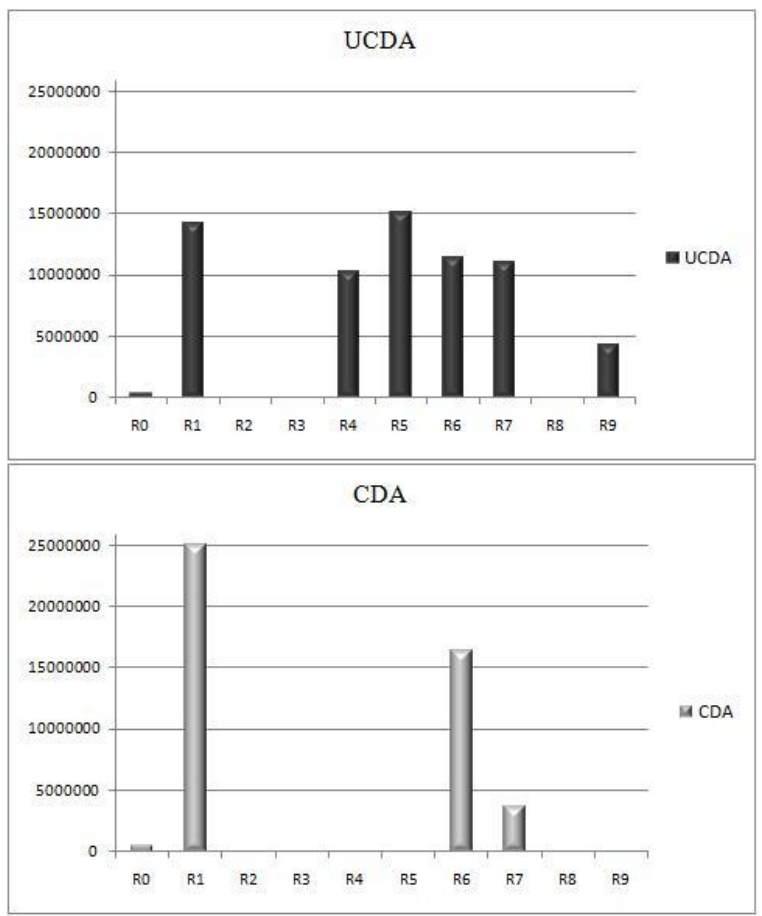

Figure 8: Greater benefit balance in UCDA for 10000 jobs

\section{CONCLUSION AND FUTURE WORK}

It is suggested in this paper, in CDA all bids- considering their high number- should be updated by the auctioneer and without a connection to the users. In additional, a method has been suggested for determining asks and bids by providers and users, which considers providers' workload and jobs' deadline. This method also provides the capability for several bids/asks in different auctions considering the condition of that auction's participants. The results of the simulation for the performance of an auctioneer shows that in the presented method - UCDA - compared to CDA:
- Jobs' success rate is higher

- Providers' benefit is greater

- The users' cost per job unit is lesser

- Resources' idleness is lesser

- The average of jobs' waiting and response time are shorter

- The Grid providers' benefit (load) balance is greater

Here bid updating is done twice in each period, it is suggested to present a solution for determining a more proper updating time, which users can increase their bids with lesser amounts.

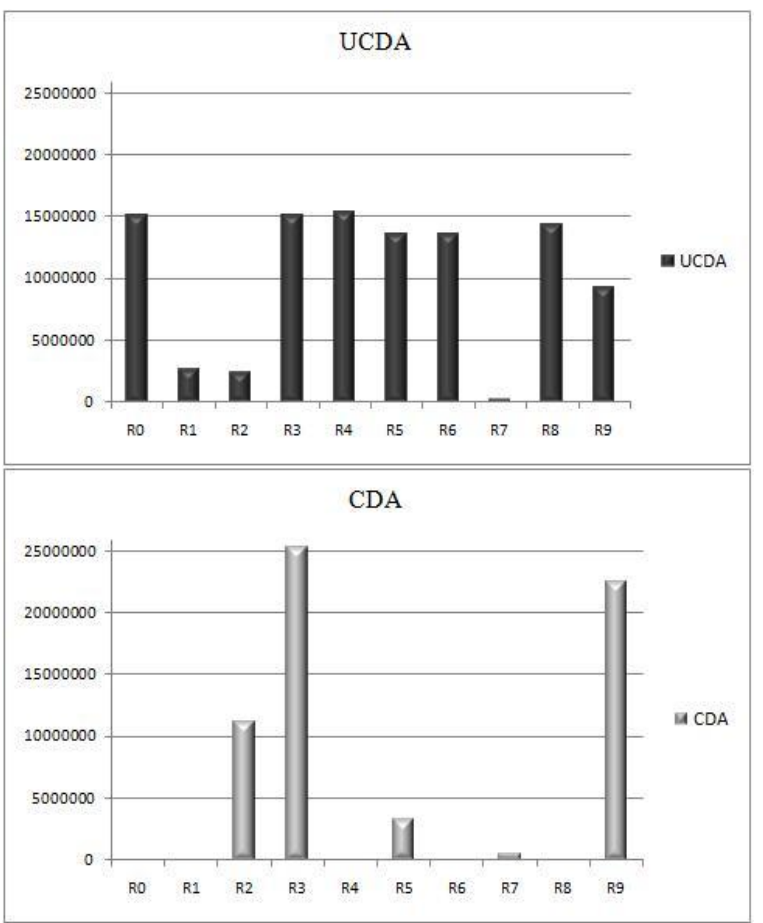

Figure 9: Greater benefit balance in UCDA for 15000 jobs

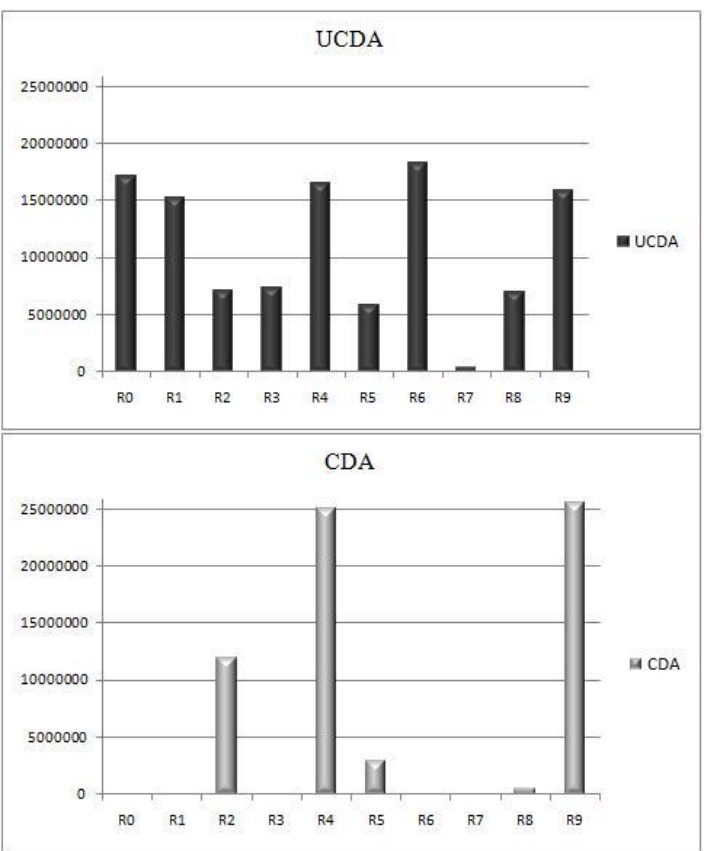

Figure 10: Greater benefit balance in UCDA for 30000 jobs 


\section{ACKNOWLEDGMENTS}

The work reported in this paper was supported by Iran Telecommunication Research Center (ITRC).

\section{REFERENCES}

[1] Buyya, R., Abramson, D., Giddy, J., Stockinger, H., "Economic Models for Resource Management and Scheduling in Grid Computing", Journal of Concurrency and Computing: Practice and Experience, pp. 1507-1542, 2002 .

[2] Buyya, R., Abramson, D., Venugopal, S., "The Grid Economy", Proc. Of the IEEE, Vol. 93, PP. 698-714, 2005.

[3] Neumann, D., Stoesser, J., "SORMA - building an open grid market for grid resource allocation", 4th international conference on Grid economics and business models Springer-Verlag Berlin, Heidelberg, 2007.

[4] Reddy P., S. R., Gupta, A., "Auction Based Resource Allocation in Grids", Distributed Computing and Networking, Springer Berlin / Heidelberg, pp. 145-156, 2006.

[5] Joita, L., Rana, O. F. Gray, W. A., Miles, J., "A Double Auction Economic Model for Grid Service", Springer Berlin / Heidelberg, pp. 409-416, 2004.

[6] Liang, Y., Fan, J., Meng, D., Di, R., “A Strategy-Proof Combinatorial Auction-Based Grid Resource Allocation System", $7^{\text {th }}$ International Conference on Algorithms and Architectures for Parallel Processing, pp. 254-266, 2007.

[7] Liang, Z., Sun, Y., Zhang, L., Dong, S., "Reverse Auction-based Grid Resource Allocation, Agent Computing and Multi-Agent Systems", Springer Berlin / Heidelberg, pp. 150-161, 2006.

[8] Vilajosana, X., Krishnaswamg, R., Marques, J., M., "Design of a configurable Auction server for Resource Allocation in Grid ", International Conference on Complex, Intelligent and Software Intensive Systems, pp. 396-401, 2009.

[9] Das, A., Grosu, D., "Combinatorial Auction-Based Protocols for Resource Allocation in Grids", 19th IEEE International Parallel and Distributed Processing Symposium (IPDPS'05), Volume 14, 2005.
[10] Kang, L., Parkes, David C., "A decentralized auction framework to promote efficient resource allocation in open computational grids", In Proceedings, Joint Workshop on The Economics of Networked Systems and Incentive-Based Computing: June 11, 2007.

[11] Garg, S.K., Venugopal, S., Buyya, R., “A Metascheduler with Auction Based Resource Allocation for Global Grids", 14th IEEE International Conference on Parallel and Distributed Systems, pp. 187-194, 2008.

[12] Izakian, H., Abraham, A., Tork Ladani, B., "An auction method for resource allocation in computational grids", Future Generation Computer Systems, Volume 26, pp. 228-235, 2010.

[13] Tan, Z., Gurd, J. R., "Market-based Grid Resource Allocation using a stable continuous Double Auction", $8^{\text {th }}$ IEEE/ACM International Conference on Grid Computing, pp. 283-290, 2007.

[14] Adabi, S., Movaghar, A., Rahmani, A., M., Beigy, H., "Market-based Grid Resource Allocation Using New Negotiation Model", Journal of Network and Computer Applications, 2012

[15] Grosu, D., Das, A., “Auction-based Resource Allocation Protocols in Grids", 16th IASTED International Conference on Parallel and Distributed Computing and Systems, 2004

[16] Kant, U., Grosu, D., "Double Auction Protocols for Resource Allocation in Grids", Proceedings of the International Conference on Information Technology: Coding and Computing, pp. 366-371, 2005.

[17] Assuncao, M., D., D., Buyya, R., “An Evaluation of Communication Demand of Auction Protocols in Grid Environments", 3rd International Workshop on Grid Economics and Business Models, pp. 1-10, 2006.

[18] Izakian, H., Ladani, B.T., Zamanifar, K., Abraham, A., Snasel, V., "A Continuous Double Auction Method for Resource Allocation in Computational Grids", IEEE Symposium on Computational Intelligence in Scheduling, 2009, pp. 29-35, 2009. 